\title{
Effect of metakaolin on chloride binding in lime-based composites
}

\author{
R. Pernicová, M. Pavlíková \& R. Černý \\ Department of Building Materials, Faculty of Civil Engineering, \\ Czech Technical University in Prague, Czech Republic
}

\begin{abstract}
The determination of chloride binding isotherms for two different types of limebased composite materials containing metakaolin is presented in this paper. The experiments are performed on small specimens with defined shape so that the effect of the porous structure is respected. Also, the experiment with crushed samples as in the original procedure by Tang and Nilsson is done for the sake of comparison and the differences analyzed. It is concluded that the chloride binding capacity of lime-based materials depends critically on the size of the sample. Therefore, the result obtained by the original Tang and Nilsson method can be considered as a certain upper limit to the real chloride binding capacity. Keywords: chloride binding isotherm, metakaolin, lime-based composites.
\end{abstract}

\section{Introduction}

Today's trend in building renovation and preservation is to use materials with defined properties and known behaviour. The demands of conservators who take care of historical monuments are that the materials for repair or innovation of plasters could have similar composition as the historical materials and they have to be applicable by the original methods, it means coated layers number and structure, the way of plaster surface treatment by striking, indentation or making it smooth.

As the chemical analyses of many plasters from historical buildings show, the past centuries external plasters that are preserved until today contain products formed by lime reaction with pozzolanic or hydraulic admixtures. In the work presented in this paper, metakaolin is used as the pozzolanic admixture in limepozzolana plaster. Application of metakaolin in lime mortars for restoration of 
renders of historical buildings is not in a contradiction with the historical reality because burnt clay containing more or less kaolinite was used in Rome and Greece 2000 years ago, as well as in further historical periods. The addition of metakaolin to lime plasters was frequently analyzed until now (e.g. [1-4]) and considered mostly a prospective way of improvement of mechanical properties.

Metakaolin is kaolinite burnt at temperatures between $500-850^{\circ} \mathrm{C}$, i.e. above the temperature of kaolinite dehydration. Quality of pozzolanic activity of particular types of metakaolin is based on the sort of raw materials used for their production, method of calcination and on granulometry of the final product. The mechanism of pozzolanic activity of metakaolin, similarly as with other pozzolanic materials, is based especially on the ability to react with calcium hydroxide. The products of this chemical reaction are CSH gels and crystallization products. The developed compounds implicate then higher strengths of lime plasters with pozzolana addition compared to pure lime plasters. These compounds are the cause of the plaster resistance against environmental conditions and in this way of the durability of these plasters.

The deterioration of porous building materials is caused in many cases by water-soluble salts. They can come into building structures from following sources:

1) Salts can be present in original building material, or they can be formed there by chemical reactions.

2) Salts come from materials used during reconstruction or renovation of building facades, for example low quality cements, impregnations etc.

3) Typical source of salts in building structures is intrusion of salty water from the grounds of buildings or capillary action of ground moisture.

4) Use of huge amounts of de-icing salts on roads and pavements in urban areas during winter period can cause metals corrosion and destroy building materials as chloride concentration in facades and masonries may be very high.

5) Due to animal excrements and microbiological organisms' activities also nitrates can be found in building structures.

6) Water-soluble salts are often transported to masonry from the soil by capillary rise of ground water.

Chlorides are potentially very dangerous for most porous building materials including renders due to their high crystallization pressures. Chloride binding capacity belongs to the most important parameters which can indicate the extent of this danger. Therefore, in this paper the main attention is paid to the chloride binding capacity of lime-metakaolin plasters as an instrument for estimating their durability.

\section{Methods for determination of ion binding isotherms}

In modelling ion transport in a porous medium, the two basic phases, namely the free phase and the bound phase, should be distinguished. Therefore, ion binding isotherms have to be determined, which express the equilibrium relation between the amount of free ions in the solution and the amount of bound ions (by both 
physisorption and chemisorption) on the pore walls in the porous medium. A simple nonlinear equilibrium ion binding isotherm was suggested by Freundlich [5]

$$
C_{b}=K \cdot C_{\mathrm{f}}^{\mathrm{m}}
$$

where $C_{b}$ is the bound ion concentration, $C_{f}$ the free ion concentration, and $K$ and $m$ are empirical parameters determined experimentally. Another simple twoparametric function was proposed by Langmuir,

$$
\frac{1}{C_{b}}=\frac{1}{k C_{\mathrm{bm}}} \cdot \frac{1}{C_{\mathrm{f}}}+\frac{1}{C_{\mathrm{bm}}}
$$

where $k$ is the adsorption constant, and $C_{b m}$ the bound chloride content at saturated monolayer adsorption.

The common method of determining the chloride binding capacity, and therefore also the chloride binding isotherms, involves dissociating the free chloride fraction from the total chloride content by analysing the pore solution squeezed out from the porous material under high pressure [6]. The total chloride content may be determined using acid-soluble extraction [7]. Tang and Nilsson [8] proposed a method for the determination of chloride adsorption isotherms based on the adsorption from solution. They calculated bound chloride content $C_{b}($ in $\mathrm{mg} / \mathrm{g}$ ) from the equation

$$
C_{b}=\frac{M_{C l} V\left(C_{0}-C_{1}\right)}{W}
$$

where $M_{C l}$ is the molar mass of chlorine, $V$ the volume of the solution (in $\mathrm{ml}$ ), $C_{0}$, $C_{I}$ the initial and equilibrium concentrations, respectively, of chloride solution (in mol/l), and $W$ the mass of the dry sample. The free chloride content $C_{f}$ (in mol/l), corresponding to the value of $C_{b}$, is equal to $C_{l}$.

By performing the experiment with different values of the initial salt concentration $C_{0}$, a point-wise function $C_{b}=C_{b}\left(C_{f}\right)$ can be obtained, which is the ion binding isotherm.

In fact, the method of Tang and Nilsson is certain idealization of the binding problem assuming that chlorides can get in direct contact with every small grain material. However, in a real specimen the interior pore surface where the material matrix is accessible to the chlorides is certainly smaller than the total surface of a crushed specimen. The chloride binding capacity can be then affected by many other factors such as the change in the porous structure and pore distribution. Therefore, the result obtained by the Tang and Nilsson method can be considered as a certain upper limit to the real chloride binding capacity. 
In this paper we have chosen a slight modification of the Tang and Nilsson method and used the specimens of more realistic dimensions. The crushed specimens were prepared as well for the sake of comparison.

\section{Materials, samples and technology of measurement}

In this paper, two lime-based plasters were studied, the first one was with metakaolin addition, VOM, and the second one was pure lime plaster for comparative purposes, VO. The mixture composition is given in Tab. 1. The amounts of particular substances are in $\mathrm{kg}$ per batch.

Table 1: $\quad$ Composition of plasters' mixtures.

\begin{tabular}{|l|l|l|l|l|}
\hline $\begin{array}{l}\text { Type of } \\
\text { Mixture }\end{array}$ & Lime CL 90 & $\begin{array}{l}\text { Sand 0/2 mm } \\
\text { Bratčice }\end{array}$ & $\begin{array}{l}\text { Metakaolin } \\
\text { Mefisto K 05 }\end{array}$ & Water \\
\hline VO & 4.80 & 14.40 & - & 4.80 \\
\hline VOM & 4.80 & 14.40 & 1.20 & 5.50 \\
\hline
\end{tabular}

The lime CL 90 was produced by limekiln "Mokrá", Czech Republic. The silica aggregates of fraction $0 / 2 \mathrm{~mm}$ were delivered by Heidelberg Cement Group, Brněnské písky Inc., affiliate Bratčice. Metakaolin MEFISTO K 05 was produced by “České lupkové závody Inc.”, Nové Strašecí. It is a highly active pozzolanic material on metakaolinite basis. MEFISTO is supposed to be used first of all as an alternative silicate binder. It can replace $5-15 \%$ of cement by weight at concrete production where it can be used instead of microsilica. It can also be utilized in the production of geopolymers. The reason for using MEFISTO in the mentioned applications is the supposed increase of compressive and flexural strength and frost resistance, decrease of water absorption and reduction of the occurrence of efflorescence. Average particle size of metakaolin is in the interval of 3 to $5 \mu \mathrm{m}$.

The samples for the measurements of chloride binding isotherms were cut from the standard prisms. Two basic forms of specimens were prepared for the

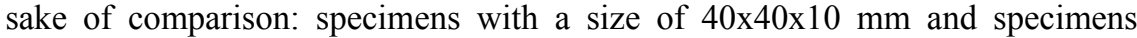
crushed in a mill into $0.06 \mathrm{~mm}$ particles. The dry samples were placed into the cups with $200 \mathrm{ml}$ chloride solution. Then they were stored in climatic chamber at $23 \pm 1^{\circ} \mathrm{C}$ to reach equilibrium. The inside solutions were analysed after six months. The concentration of chlorides was determined using the $\mathrm{pH} / \mathrm{ION} 740$ device with ion selective electrodes (ISE) to obtain the bound chloride content. The most important step is to calibrate chloride selective electrodes with different known chlorides concentrations diluted from standard solution. The amount of chloride ions in liquid samples can be then easily and rapidly determined using chloride selective electrodes. The ISE cell is immersed into measured solution and chloride concentration in $\mathrm{mg} / \mathrm{l}$ solution is shown on the display of the $\mathrm{pH} / \mathrm{Ion}$ measuring device. From the obtained data the bound chloride content was calculated and chloride binding isotherms were plotted. 
The chloride contents in the measured materials themselves were obtained by leaching granulated gravel in boiling water. Chloride content measured in this way using the chloride selective electrode was $0.2 \mathrm{mg} / \mathrm{l}$ in both cases.

\section{Experimental results and discussion}

Firstly, bulk density, as a fundamental physical characteristic of the material, was determined on the vacuum saturation principle using Archimedes' weight. Bulk densities of tested materials were $1650 \mathrm{~kg} \mathrm{~m}^{-3}$ in the case of lime plaster and $1670 \mathrm{~kg} \mathrm{~m}^{-3}$ for lime plaster with metakaolin addition.

Fig. 1 shows chloride binding isotherms of lime plaster, VO, and lime plaster with metakaolin addition, VOM, measured by modified Tang and Nilsson adsorption method, i.e., for samples with defined shape. Clearly, the chloride binding capacity of both studied materials was quite high. The addition of metakaolin caused up to $30 \%$ increase of chloride binding capacity which was due to the formation of CSH structures.

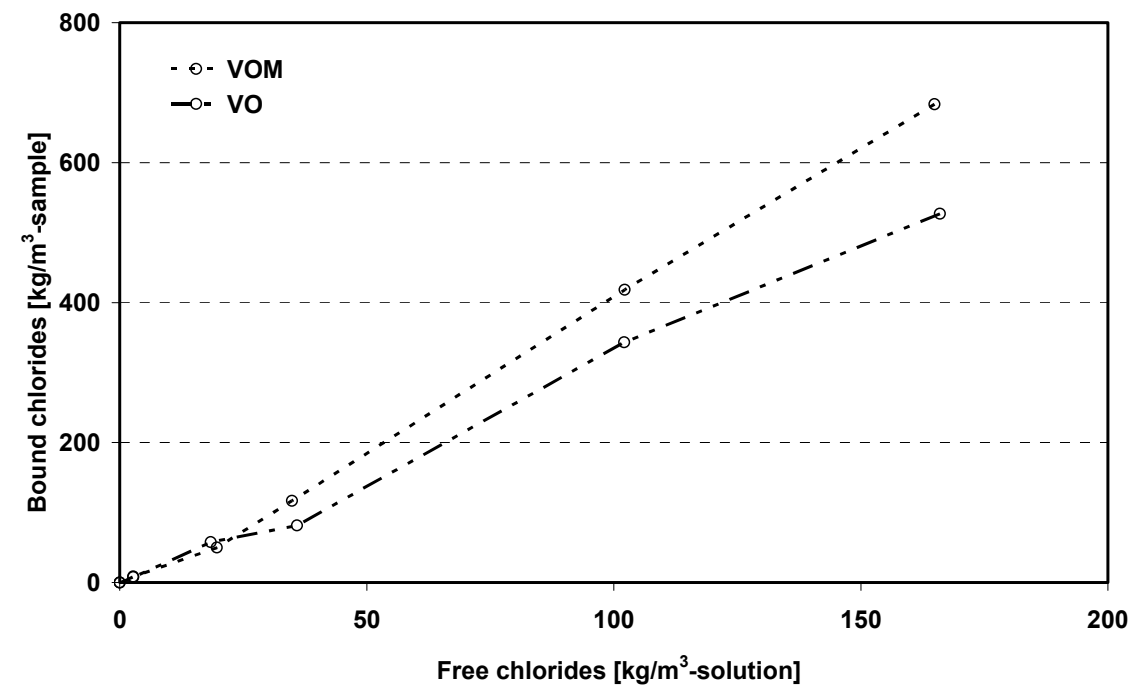

Figure 1: Chloride binding isotherms of lime-based composites measured by modified adsorption method.

Fig. 2 presents chloride binding isotherms measured with crushed particles of both studied lime-based composites as in the original Tang and Nilsson [8] procedure. Metakaolin addition led to higher binding capacity in this case as well.

Figs. 3 and 4 compare chloride binding isotherms measured for finitedimension samples and crushed particles. The differences were for both tested materials mostly (except for one measuring point) not very high, up to $15 \%$ only. 


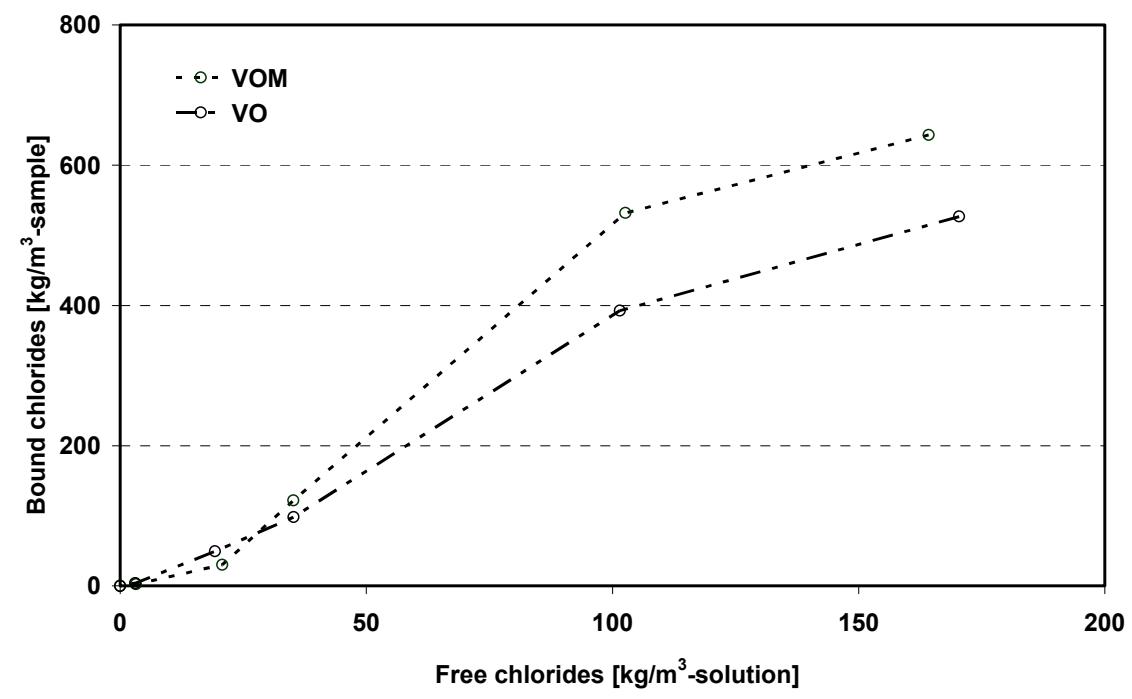

Figure 2: Chloride binding isotherms of lime-based composites measured for crushed particles.

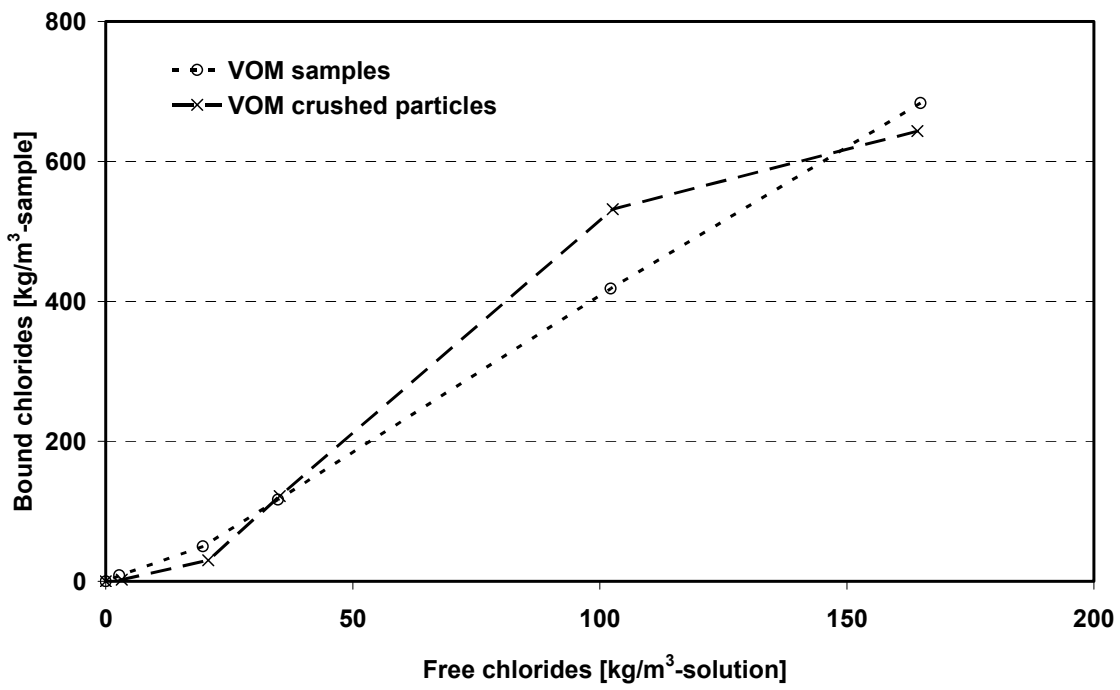

Figure 3: Comparison of chloride binding isotherms of finite-dimension samples and crushed particles for lime-based plaster with metakaolin addition.

Fig. 5 presents a comparison of chloride binding isotherm of lime plaster with that of gypsum. The data for bound chlorides are recalculated to mg of chlorides per $g$ of sample to exclude the effect of different bulk densities. The measured 
results show that lime-based composites can bind much higher amount of chlorides than even gypsum which already possesses relatively high chloride binding capacity.

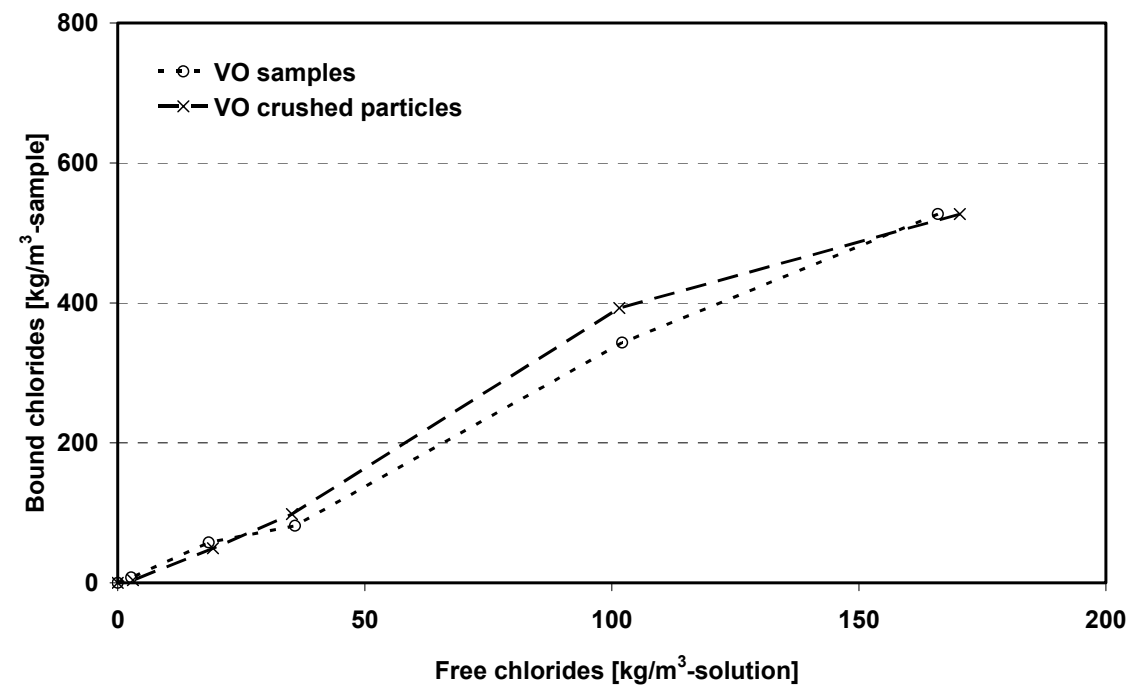

Figure 4: Comparison of chloride binding isotherms of finite-dimension samples and crushed particles for lime plaster.

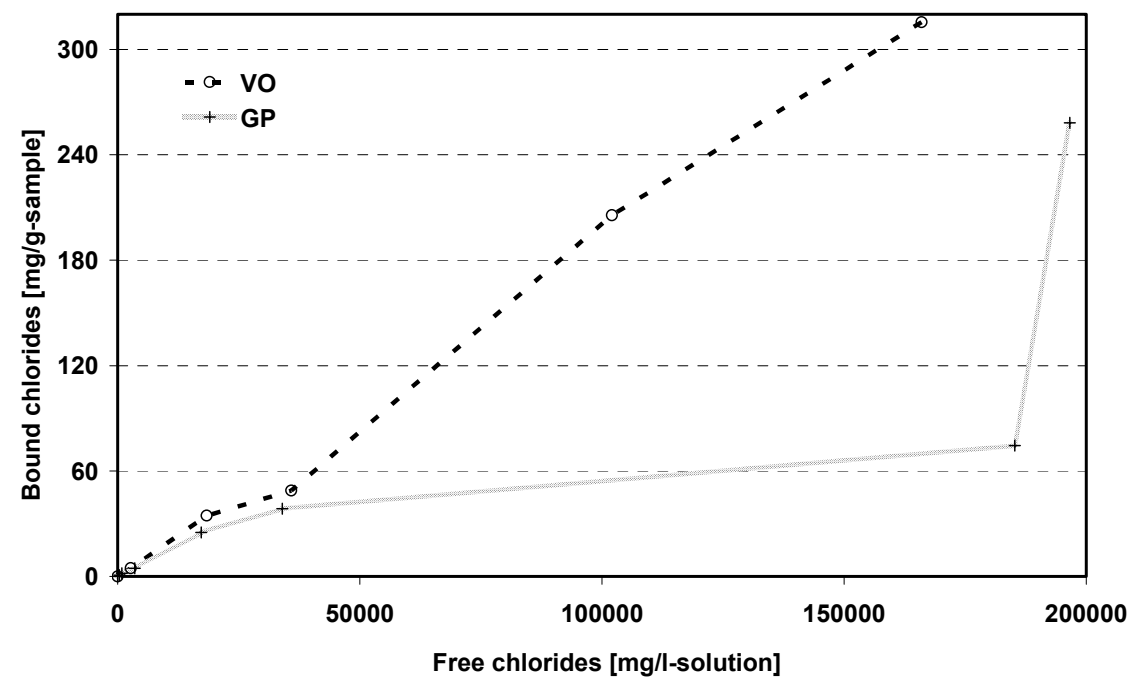

Figure 5: Comparison between chloride binding isotherm of gypsum and lime plaster. 
In an analysis of the uncertainty of measurements in this paper, it should be noted first that achieving equilibrium for even rather small but not crushed samples is a long-term problem and the experiment takes usually several months. The measuring conditions during this time are supposed to remain stable. The measuring error in the determination of chloride concentration can be considered in our experiments as $10 \%$ or less because no interference effects of other present ions in measured solutions appeared.

\section{Conclusions}

The chloride binding isotherms of two different types of lime-based plasters were determined in the paper. The materials were tested in the form of samples with realistic dimensions and crushed particles. Experimental results showed that lime plaster with metakaolin addition had up to $30 \%$ higher chloride binding capacity than reference lime plaster which itself was quite high, much higher than for instance of gypsum. This may lead to relatively fast filling of the pore space of lime-based plasters by chlorides they are exposed to. On one hand this may be advantageous because for instance chlorides can be extracted from the underlying structure and transported to the render; for historical building this feature can be quite useful. On the other, the hygric properties of plasters with higher amount of bound chlorides can be changed significantly. In particular, there is an open question how the water vapour diffusion capability of the plaster will be affected. Another question is how the high amount of bound chlorides will affect the freeze/thaw resistance. These issues will be subject of further investigations.

\section{Acknowledgement}

This research was supported by the Czech Science Foundation, under project No. 103/06/0031.

\section{References}

[1] Cabrera, J., Rojas, M. F., Mechanism of hydration of the metakaolin-lime water system, the hydration phases of metakaolin-lime-water systems, Cement and Cement and Concrete Research, 31, pp. 177-182, 2001.

[2] Rojas, M. F., Cabrera, J., The effect of temperature on the hydration rate and stability of the hydration phases of metakaolin-lime-water systems, Cement and Concrete Research, 32, pp. 133-138, 2002.

[3] Rojas, M. F., Sánchez de Rojas, M. I., The effect of high curing temperature on the reaction kinetics in MK-lime and MK-blended cement matrices at $60{ }^{\circ} \mathrm{C}$, Cement and Concrete Research, 33, pp. 643-649, 2003.

[4] Schwarzmann A., The effect of dehydroxylation/amorphization degree on pozzolanic activity of kaolinite, Cement and Concrete Research, 33, pp. 405-416, 2003. 
[5] Freundlich, C.G.L., Colloid and Capillary Chemistry. Metheun, London 1926.

[6] Dhir, R.K., Jones, M.R., Ahmed, H.E.H., Determination of total and soluble chlorides in concrete. Cement and Concrete Research, 20, pp. 579$590,1990$.

[7] Glass, G.K., Buenfeld, N.R., The determination of chloride binding relationships. In: Proceedings of the RILEM International Workshop Chloride Penetration into Concrete, edited by Nilsson, L.O. and Ollivier, J.P. RILEM, St-Remy-les-Chevreuse, 1997, pp. 3-9.

[8] Tang, L., Nilsson, L.O., Chloride binding capacity and binding isotherms of OPC pastes and mortars. Cement and Concrete Research, 23, pp. 247$253,1993$. 